\title{
Unknown Outcome
}

National Cancer Institute

\section{Source}

National Cancer Institute. Unknown Outcome. NCI Thesaurus. Code C159895.

The outcome is unknown. 